\title{
Employment status, difficulties at work and quality of life in inflammatory bowel disease patients
}

\author{
Angela G.E.M. De Boer ${ }^{a}$, Floor Bennebroek Evertsz'b ${ }^{\prime b}$ Pieter C. Stokkers ${ }^{c}$, Claudia L. Bockting ${ }^{\mathrm{d}, \mathrm{e}}$, \\ Robert Sanderman ${ }^{f}$, Daniel W. Hommes ${ }^{g}$, Mirjam A.G. Sprangers ${ }^{b}$ and Monique H.W. Frings-Dresen ${ }^{a}$
}

\begin{abstract}
Objectives To assess employment status, difficulties at work and sick leave in inflammatory bowel disease (IBD) patients and their relation with sociodemographic and clinical factors, quality of life (QoL), and anxiety and depression.

Materials and methods IBD patients attending an IBD outpatients' clinic received self-report questionnaires on employment status, IBD-related difficulties at work and sick leave (Trimbos/iMTA questionnaire for Costs associated with Psychiatric Illness), sociodemographic factors, QoL (Inflammatory Bowel Disease Questionnaire and 12-item Short-form Health Survey) and anxiety and depression (Hospital Anxiety and Depression Scale). Disease activity was assessed by their gastroenterologist. Associations between paid employment and sick leave with sociodemographic and clinical factors, QoL and anxiety and depression were assessed by regression analyses.

Results In total, 202 IBD patients of working age, with a mean age of 41 years, participated; 63\% had Crohn's disease and $37 \%$ had ulcerative colitis, and 57\% were women and 19\% had active disease. In all, $123(61 \%)$ patients were in paid employment, of whom 31 (25\%) were on sick leave, whereas $46(23 \%)$ received a disability pension. Concentration problems (72\%), low working pace $(78 \%)$ and delayed work production $(50 \%)$ were the most prevalent IBD-related work difficulties. IBD patients without paid employment were older and more often women, with active disease, lower QoL and higher anxiety and depression rates. Sick leave was associated with lower QoL and higher anxiety and depression rates.

Conclusion More than half of IBD patients were in paid employment, whereas almost a quarter was receiving a disability pension. A large majority experienced work difficulties. Having no paid employment was associated with poorer QoL and more anxiety and depression symptomatology. Eur J Gastroenterol Hepatol 28:1130-1136

Copyright (c) 2016 Wolters Kluwer Health, Inc. All rights reserved.
\end{abstract}

\section{Introduction}

The prevalence of inflammatory bowel disease (IBD) is relatively high in Europe, with 505 persons affected with ulcerative colitis (UC) per 100000 persons and 322 persons per 100000 persons affected by Crohn's disease (CD). Many IBD patients develop intestinal or extraintestinal complications and experience adverse treatment effects [1]. The potentially incapacitating disease course can represent a heavy burden for patients, impacting every aspect of the patient's quality of life (QoL) [1-3].

Typically those in the young working-age population are affected [4]. The majority of patients with IBD experience physical, emotional and social problems such

\footnotetext{
European Journal of Gastroenterology \& Hepatology 2016, 28:1130-1136

Keywords: anxiety, concentration, depression, disability pension, employment, inflammatory bowel disease, quality of life, sick leave, work

${ }^{a}$ Coronel Institute of Occupational Health, 'Department of Medical Psychology, Academic Medical Center, 'Department of Gastroenterology, Sint Lucas Andreas Hospital, Amsterdam, dDepartment of Clinical and Health Psychology, Utrecht University, Utrecht, e Department of Clinical Psychology, 'Department of Health Sciences, University Medical Center Groningen, University of Groningen, Groningen, The Netherlands and 9Department of Medicine, Center for Inflammatory Bowel Diseases, University of California Los Angeles, Los Angeles, California, USA

Correspondence to Angela G.E.M. De Boer, PhD, Coronel Institute of Occupational Health, Academic Medical Center, PO Box 22700, 1100 DE Amsterdam, The Netherlands

Tel: +3120 566 5323; fax: +3120 697 7161; e-mail: a.g.deboer@amc.nl

Received 4 March 2016 Accepted 11 May 2016
}

as fatigue, pain, diarrhoea, diminished cognitive functioning, embarrassment and anxiety or depression, which may result in limitations in social activities including employment [3]. An important consequence is, therefore, a reduced ability to work that can have a severe negative effect on patients' health-related QoL [5] and that leaves IBD patients at risk of leaving the labour force [1].

High work disability rates have been reported for IBD patients compared with the general population $[5,6]$, with a six- to nine-fold increased risk of disability pension [7]. In a longitudinal study, it was reported that IBD patients experienced an increased risk for disability pension compared with the general population even 10 years after disease onset [8]. Disease severity was found to be the most important predictor of work disability $[6,8,9]$.

In addition, a large proportion of the societal costs associated with IBD are indirect costs that are associated with sick leave and early retirement [8]. In the USA, IBD patients have higher probabilities of missing time from work and missing workdays than those without IBD [10]. Work productivity is significantly impaired in IBD patients and is associated with high presenteeism and productivity loss [7].

Because earlier studies have primarily reported on work outcomes such as sick leave data, little is known about the difficulties that IBD patients themselves experience while they are working. Furthermore, few data are available on employment status, IBD-related difficulties at work, sick leave and how they are associated with disease factors and 
QoL outcomes. One Spanish study found that higher subjective work disability scores in CD patients were correlated to lower QoL scores and higher number of sick leave days [11].

Employees with a chronic physical condition such as IBD may be hampered in job performance and employment because of physical or cognitive limitations, pain, fatigue or because medical treatment interferes with work [12]. Unemployment is associated with anxiety and depression among IBD patients [13]. Information on IBDrelated work difficulties and disease activity, QoL, anxiety and depression outcomes could thus help clinicians in detecting those IBD patients at risk for unfavourable employment outcomes very early in the treatment process and, if indicated, refer them to occupational care.

The aims of this study are to assess (a) the employment status of IBD patients and IBD-related difficulties at work; (b) the relationship between the work-related outcomes employment status and sick leave and sociodemographic factors, clinical factors, IBD-related difficulties at work, QoL, anxiety and depressive symptoms; and (c) healthcare utilization and its associations with work-related factors and QoL.

\section{Materials and methods}

\section{Patients and procedures}

Consecutive IBD patients who attended the IBD outpatients' clinic of the University Hospital of Amsterdam, a top referral centre, were invited by their treating gastroenterologist to participate in the study during their hospital visit. All patients had IBD, diagnosed by radiography of the bowel and colon, colonoscopy and endoscopic biopsy. Patients received an informed consent form to be signed at home along with a set of self-report questionnaires by mail. They were asked to return the completed questionnaire using a prestamped envelope within 2 weeks. Those who did not respond received a digital reminder once. The Institutional Review Board (IRB) of the Medical Ethics Committee of the Academic Medical Center considered that the Medical Research Involving Human Subjects Act (WMO) did not apply to the study and that an official approval of this study by the Committee was not required.

\section{Measures}

\section{Employment status and difficulties at work}

All measures of employment were assessed using the Trimbos/iMTA questionnaire for Costs associated with Psychiatric Illness (TiC-P) as the items were relevant for IBD [14]. Employment status was assessed as having a paid job (yes/no). Having no paid job was measured as either being unemployed/having voluntary work, being work-disabled, being a student, being a housewife/homemaker or being an early pensioner. IBD-related work difficulties in the past month including concentration problems, having to postpone work, having to work slower and difficulties making decisions were assessed with the TiC-P on a four-point scale, with lower scores indicating fewer problems. In addition, for IBD patients with a paid job, the extent to which IBD interfered with work (not, somewhat, very), work presenteeism in the past month (number of days at work but actually experiencing health problems) and work efficiency in the past 2 weeks (scale 1-10 from very inefficient to as efficient as normal) were assessed. Furthermore, number of working hours per week, number of working days per week, occupation, sick leave days in the past 2 weeks and income were assessed.

Sociodemographic factors, clinical factors and healthcare utilization

Sociodemographic variables included sex, age, marital status and education (primary education, secondary education, higher education, college/university). Clinical variables were assessed by the gastroenterologist and included disease type (CD, UC), disease activity (yes/no), number of (IBD-related) operations, duration of disease in years and undergoing an ileostomy (yes/no). The Harvey Bradshaw Index [15] was used to assess disease activity of CD patients and the Truelove and Witts Index [16] assessed disease activity of UC patients. A score of higher than 4 on both scales is indicative of disease activity. Healthcare utilization was assessed with the TIC-P completed by the patients [14] and included number of visits to the occupational physician, general practitioner (GP), physiotherapist, social worker or outpatient visits to a specialist during the previous 4 weeks.

\section{Quality of life}

Perceived QoL was measured using the 12-item Short-form Health Survey (SF-12), an abbreviated version of the SF-36 [17]. This generic instrument focuses on general health, physical functioning, mental health, role limitations because of vitality, physical and mental health, emotional problems, pain, vitality and social function. Two dimension scores are derived from the item scores: the Physical Component Summary (PCS) and the Mental Component Summary (MCS). Higher SF-12 scores indicate a higher level of QoL [18]. The Dutch norm score is 49.4 for PCS and 51.6 for MCS $[17,18]$.

Disease-specific QoL was measured using the Inflammatory Bowel Disease Questionnaire (IBDQ) [19] consisting of 32 items, which are scored on a five-point Likert scale. The questions are grouped into four subscales: bowel symptoms, systemic symptoms, emotional function and social function, and a total score is calculated [3]. The IBDQ is a reliable and (cross-culturally) valid instrument $[3,20]$ with a potential score range of 32 to 224, with higher scores indicating better disease-specific QoL. Anxiety and depression were measured using the Hospital Anxiety and Depression Scale (HADS). The scale contains 14 items consisting of HADS-A (anxiety, seven questions) and HADS-D (depression, seven questions) subscales [21]. All items are rated on a four-point scale (0-3), with higher scores indicating higher levels of depression and/or anxiety. A score of greater than or equal to 8 on either subscale indicates a possible psychiatric disorder (anxiety and/or depressive disorder).

\section{Statistical analyses}

Patients more than or equal to 65 years were excluded from analyses because of the legal retirement age of 
65 years in the Netherlands. Employment status, IBDrelated work problems, sick leave in the last 2 weeks, sociodemographic and clinical factors, QoL, anxiety and depression scores and healthcare utilization are described. Comparison of QoL SF-12 scores, QoL IBDQ scores and anxiety and depression HADS scores between IBD patients with a paid job and those without a paid job were analysed by analysis of covariance corrected for age and sex. Associations between sick leave (yes/no) with sociodemographic and clinical factors, QoL, anxiety and depression, presenteeism, work efficiency and other work problems were analysed by correlations/crosstabs and (logistic) regression analyses. Associations between disease activity (yes/no) and presenteeism, work efficiency, concentration problems at work and working slower were analysed using nonparametric tests. Associations of healthcare utilization (number of visits to the occupational physician, GP, physiotherapist, social worker or outpatient visits to a specialist) with sociodemographic and clinical factors, QoL, anxiety and depression and presenteeism, work efficiency and other work problems were analysed by multiple regression analyses.

\section{Results}

We sent self-report questionnaires to 292 consecutive IBD patients who had attended the IBD Outpatients' Clinic of the University Hospital of Amsterdam. In all, $231 \mathrm{com}-$ pleted questionnaires were returned $(79 \%$ response rate). Of these 231 IBD patients, 202 IBD patients $(87.4 \%)$ were less than 65 years of age. The mean age of these 202 IBD patients was 41 years (range 18-64); 116 patients (57\%) were women and most patients were married $(70 \%)$. Twothirds of the patients had CD and one-third had UC, with $19 \%$ of the patients in active disease and $8 \%$ with ileostomy (Table 1).

\section{Employment status, sick leave and work difficulties}

Table 2 shows that 123 IBD patients were in paid employment $(61 \%)$ and 46 IBD patients $(23 \%)$ were

\begin{tabular}{lc}
\hline Table 1. Sociodemographic and clinical patient characteristics \\
\hline$N=202$ patients $^{\text {a }}$ & $n(\%)$ \\
\hline Age [mean (range, SD)] (years) & $41(18-64,12)$ \\
Disease duration [mean (range, SD)] (years) & $15(0-36,9.3)$ \\
Female & $116(57)$ \\
Marital status & \\
$\quad$ Married, living together & $141(70)$ \\
Single, divorced, widowed & $61(30)$ \\
Disease & \\
Crohn's disease & $128(63)$ \\
Ulcerative colitis & $74(37)$ \\
Active disease & \\
Yes & $31(19)$ \\
No & $134(81)$ \\
Education & $41(22)$ \\
Primary education & $63(33)$ \\
Secondary education & $18(10)$ \\
Higher education & $66(35)$ \\
College/university & $17(8)$ \\
lleostomy (yes) & $110(55)$ \\
Number of operations & $91(45)$ \\
0 &
\end{tabular}

${ }^{a}$ As a result of missing data, not all outcomes add up to $N=202$ patients.

\begin{tabular}{lc}
\hline Table 2. Employment outcomes & \\
\hline$N=202$ patients & $n(\%)$ \\
\hline Employment status & \\
Paid employment & $123(61)$ \\
Unemployment & $1(0.5)$ \\
Housewife/homemaker & $12(6)$ \\
Student & $11(5)$ \\
Disability pension/work disabled & $46(23)$ \\
Early retirement $(<65$ years) & $9(5)$ \\
If in paid employment (N=123) & \\
Sick leave in the last 2 weeks (yes) & $31(25)$ \\
Hours working per week [mean (SD)] & $31(10.2)$ \\
Work hindered by health problems in the last 2 weeks & \\
No & $58(52)$ \\
Somewhat & $45(40)$ \\
Very & $8(7)$ \\
At work, but concentration problems & $14(28)$ \\
Never & $24(48)$ \\
Sometimes & $12(24)$ \\
Often & \\
At work, but had to work slower & $11(22)$ \\
Never & $20(41)$ \\
Sometimes & $15(31)$ \\
Often & $3(6)$ \\
Almost always & \\
At work, but had to seclude oneself & $23(46)$ \\
Never & $17(34)$ \\
Sometimes & $8(16)$ \\
Often & $2(4)$ \\
Almost always & $30(60)$ \\
At work, but difficulty making decisions & $15(31)$ \\
Never & $4(8)$ \\
Sometimes & \\
Often & $25(50)$ \\
At work, but work delayed & $24(48)$ \\
Never & $1(2)$ \\
Sometimes & $36(72)$ \\
Often & $12(24)$ \\
At work, but work had to be done by others & $1(2)$ \\
Never & $1(2)$ \\
Sometimes & \\
Often & \\
Almost always & \\
\hline As a rest of mising & \\
\hline
\end{tabular}

${ }^{a}$ As a result of missing data, not all outcomes add up to $N=123$ patients.

receiving a disability pension. On average, employed IBD patients worked $31 \mathrm{~h}$ per week. Of the 123 employed patients, $25 \%$ had been on sick leave in the last 2 weeks and almost half $(47 \%)$ of the patients reported that their work had been hindered by health problems in the last 2 weeks. Of the patients who were actually at work, $72 \%$ experienced concentration problems, $78 \%$ had to work slower than normal, $54 \%$ had to seclude themselves from their colleagues and $40 \%$ had difficulties making decisions. Furthermore, $50 \%$ were at work, but their work production was delayed, whereas for $28 \%$ of the patients, their work had to be done by others.

\section{Paid employment and associations with other factors}

Sociodemographic and disease-related factors, and QoL, anxiety and depression scores for IBD patients with and without paid employment are shown in Table 3.

IBD patients without paid employment were older $(47$ vs. 40 years, $P<0.001)$ and more often women (68 vs. $49 \%, P=0.02)$ than those in paid employment. Active disease was more often present in IBD patients without paid employment compared with those in paid employment ( 28 vs. $13 \%, P=0.03$ ), but no differences in disease duration were found (14 vs. 17 years, $P=0.41$ ). 
Table 3. Differences in sociodemographic, disease-related, QoL, anxiety and depression variables between IBD patients with and without paid employment

\begin{tabular}{|c|c|c|c|c|}
\hline & All patients $(N=202)^{a}$ & No paid employment ${ }^{\mathrm{b}}(N=79)^{\mathrm{a}}$ & Paid employment $(N=123)^{a}$ & $P$-value \\
\hline Age (years) & 41 & 47 & 40 & $<0.001$ \\
\hline Sex (female) (\%) & 57 & 68 & 49 & 0.02 \\
\hline Disease activity (yes) (\%) & 19 & 28 & 13 & 0.03 \\
\hline Disease duration (years) & 15 & 17 & 14 & 0.41 \\
\hline \multicolumn{5}{|l|}{ QoL: SF-12 [mean (SD)] } \\
\hline Physical Component Score & $42(11)$ & $36(10)$ & $45(10)$ & $<0.001$ \\
\hline Mental Component Score & $47(9)$ & $45(9)$ & $47(9)$ & 0.33 \\
\hline \multicolumn{5}{|l|}{ QoL: IBDQ [mean (SD)] } \\
\hline IBDQ total & $169(35)$ & $155(34)$ & 174 (33) & $<0.001$ \\
\hline IBDQ bowel & $53(11)$ & $50(11)$ & $55(11)$ & 0.004 \\
\hline IBDQ systemic & $23(7)$ & $19(7)$ & $24(6)$ & $<0.001$ \\
\hline IBDQ emotional & $64(13)$ & $60(14)$ & $65(13)$ & 0.004 \\
\hline IBDQ social & $28(7)$ & $25(7)$ & $29(7)$ & $<0.001$ \\
\hline Anxiety: HADS [mean (SD)] & $6.1(4)$ & $7.9(4)$ & $5.2(4)$ & $<0.001$ \\
\hline Anxiety (HADS $\geq 8)[n(\%)]$ & 73 (37) & $26(48)$ & $37(30)$ & 0.02 \\
\hline Depression: HADS [mean (SD)] & $4.7(4)$ & $7.2(4)$ & $3.5(4)$ & $<0.001$ \\
\hline Depression (HADS $\geq 8)[n(\%)]$ & $51(26)$ & $22(41)$ & $22(19)$ & 0.002 \\
\hline
\end{tabular}

Age-adjusted and sex-adjusted analyses.

HADS, Hospital Anxiety and Depression Scale; IBD, inflammatory bowel disease; IBDQ, Inflammatory Bowel Disease Questionnaire; QoL, quality of life; SF-12, 12-item Short-form Health Survey.

${ }^{a}$ As a result of missing data, not all outcomes are for $N=202$ patients.

${ }^{b}$ No paid employment including disability pension, homemaking, study, early retirement and unemployment benefit.

Results of the age-adjusted and sex-adjusted analysis of covariance of QoL outcomes showed that the PCS scores ( 36 vs. $45, P<0.001$ ), the IBDQ total scores ( 155 vs. 174 , $P<0.001$ ) and all IBDQ subscale scores (bowel, systemic, emotional and social; all $P<0.005$ ) were significantly lower for IBD patients without paid employment compared with those with paid employment, but the MCS scores did not differ (45 vs. $47, P=0.33$ ).

The mean HADS anxiety and depression scores were 6.1 and 4.7 , respectively, with $37 \%$ of IBD patients having high anxiety scores indicative of a possible psychiatric disorder of anxiety and $26 \%$ having high depression scores indicative of a possible psychiatric disorder of depression. IBD patients without paid employment were found to have higher scores on anxiety and depression compared with those with paid employment (7.9 vs. 5.2, $P<0.001$, and 7.2 vs. $3.5, P<0.001$, respectively) as indicated by age-adjusted and sex-adjusted analysis of covariance. Furthermore, IBD patients without paid employment showed statistically significantly more often HADS scores that are indicative of a possible psychiatric disorder of anxiety and depression compared with IBD patients with paid employment ( 48 vs. $30 \% ; P=0.02$, and 41 vs. $19 \% ; P=0.002$, respectively).

\section{Sick leave and associations with other factors}

Sick leave data of the 123 employed IBD patients are shown in Table 4 . In the last 2 weeks, $31(25 \%)$ of the employed IBD patients had been on sick leave. No differences in age $(P=0.24)$, sex $(P=0.38)$, disease activity $(P=0.64)$ or disease duration $(P=0.46)$ were found between employed IBD patients who had been on sick leave and those who had not. IBD patients on sick leave reported statistically significantly lower scores with respect to SF-12 physical $(P<00.001)$ and mental health $(P=0.005)$ scores, IBDQ total $(P<0.001)$, bowel $(P<0.001)$, systemic $(P<0.001)$, emotional $(P=0.001)$ and social scores $(P<0.001)$, and HADS anxiety $(P=00.02)$ and depression scores $(P=0.04)$ compared
Table 4. Differences in sociodemographic, disease-related, QoL and work-related factors between employed IBD patients who were and were not on sick leave in the last 2 weeks

\begin{tabular}{|c|c|c|c|}
\hline$N=123^{\mathrm{a}}$ patients & $\begin{array}{l}\text { Sick leave in the } \\
\text { last } 2 \text { weeks } \\
(N=31)\end{array}$ & $\begin{array}{l}\text { No sick leave in the } \\
\text { last } 2 \text { weeks } \\
(N=94)\end{array}$ & $P$-value \\
\hline Age (years) & 38 & 40 & 0.24 \\
\hline Sex (female) (\%) & 55 & 46 & 0.38 \\
\hline Disease activity (yes) & 16 & 12 & 0.64 \\
\hline Disease duration (years) & 14 & 15 & 0.46 \\
\hline \multicolumn{4}{|l|}{ QoL: SF-12 scores } \\
\hline $\begin{array}{l}\text { Physical Component } \\
\text { Score }\end{array}$ & 38 & 47 & $<0.001$ \\
\hline $\begin{array}{l}\text { Mental Component } \\
\text { Score }\end{array}$ & 43 & 48 & 0.005 \\
\hline \multicolumn{4}{|l|}{ QoL: IBDQ scores } \\
\hline IBDQ total & 150 & 180 & $<0.001$ \\
\hline IBDQ bowel & 48 & 57 & $<0.001$ \\
\hline IBDQ systemic & 20 & 25 & $<0.001$ \\
\hline IBDQ emotional & 57 & 66 & 0.001 \\
\hline IBDQ social & 24 & 31 & $<0.001$ \\
\hline Anxiety: HADS & 7.4 & 5.2 & 0.02 \\
\hline Depression: HADS & 5.3 & 3.7 & 0.04 \\
\hline Presenteeism (days) & 4.9 & 4.6 & 0.80 \\
\hline Work efficiency & 6.0 & 6.8 & 0.16 \\
\hline Concentration problems & 2.0 & 1.9 & 0.79 \\
\hline Work slower & 2.4 & 2.1 & 0.19 \\
\hline
\end{tabular}

HADS, Hospital Anxiety and Depression Scale; IBD, inflammatory bowel disease; IBDQ, Inflammatory Bowel Disease Questionnaire; QoL, quality of life; SF-12, 12-item Short-form Health Survey.

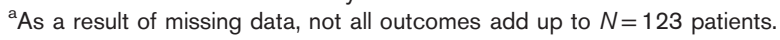

with those not on sick leave. No differences were found between the groups on the work-related outcomes of presenteeism $(P=0.80)$, work efficiency $(P=0.16)$, concentration problems $(P=0.79)$ and working slower $(P=0.19)$. No differences were found between IBD patients with and without disease activity in presenteeism $(P=0.62)$, work efficiency $(P=0.15)$ and problems concentrating at work $(P=0.57)$. However, patients with active disease more often reported working slower than patients with no active disease $(P=0.03)$. 


\section{Healthcare utilization and associations with other factors}

The occupational physician was visited by $10 \%$ of IBD patients, whereas a GP was visited by $45 \%$ of the IBD patients in the last 4 weeks (Table 5). A physiotherapist was visited by $13 \%$, whereas $5 \%$ of the IBD patients consulted a social worker. Most IBD patients $(51 \%)$ paid one visit to a specialist in the last 4 weeks.

Number of visits to the occupational physician was associated with sick leave in the last 2 weeks $(P<0.0001)$, the IBDQ total score $(P=0.03)$, emotional scale $(P=0.003)$, social scale $(P=0.02)$, bowel symptoms $(P=0.02)$, HADS anxiety $(P=0.006)$ and SF-12 mental score $(P=0.10)$ as indicated by multiple regression analysis.

The number of visits to the GP was associated with the IBDQ total score $(P=0.04)$ social scale $(P=0.02)$, systemic symptoms $(P=0.02)$ and the SF-12 physical scores $(P=0.04)$.

Number of visits to the medical specialist was associated with IBDQ total score $(P=0.003)$, emotional scale $(P=0.04)$, social scale $(P<0.001)$, systemic symptoms $(P=0.02)$ and the SF-12 physical scores $(P<0.0001)$. Visits to the physiotherapist were associated with sick leave $(P=0.001)$, IBDQ total score $(P=0.02)$, social scale $(P=0.008)$, systemic symptoms $(P=0.01)$ and the SF-12 physical scores $(P=0.001)$. Visits to the social worker were not related to any of the work-related or QoL factors.

\section{Discussion}

The aim of this study was to assess the employment status, difficulties at work and sick leave of IBD patients to evaluate the relationship between employment status and sick leave, and sociodemographic and clinical factors, QoL, anxiety and depressive symptoms, and to assess healthcare utilization and its associations with workrelated factors and QoL. Results showed that IBD exerts

\begin{tabular}{lc}
\hline \multicolumn{2}{l}{ Table 5. Healthcare utilization: mean number of visits in the last 4 weeks } \\
\hline$N=202$ patients $^{\mathrm{a}}$ & $n(\%)$ \\
\hline Occupational physician & \\
0 & $181(90)$ \\
1 & $15(7)$ \\
2 & $3(2)$ \\
3 or more & $3(2)$ \\
General practitioner & \\
0 & $111(55)$ \\
1 & $59(29)$ \\
2 & $20(10)$ \\
3 or more & $12(6)$ \\
Physiotherapist & \\
0 & $175(87)$ \\
1 & $4(2)$ \\
2 & $6(3)$ \\
3 or more & $17(8)$ \\
Social worker & \\
0 & $192(95)$ \\
1 & $6(3)$ \\
2 & $3(2)$ \\
3 or more & $1(1)$ \\
Outpatient visits to a specialist & \\
0 & $43(21)$ \\
1 & $102(51)$ \\
2 & $35(17)$ \\
3 or more & $22(11)$ \\
\hline
\end{tabular}

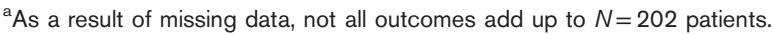

a detrimental effect on the employment situation of IBD patients and that most working patients experienced work difficulties. Unfavourable work outcomes such as no paid employment and being on sick leave were associated with lower QoL and higher anxiety and depression scores. The number of visits to the occupational physician was associated with sick leave, QoL and anxiety.

\section{Strengths and limitations}

A strength of this study is the collection of data on work difficulties as experienced by the IBD patients themselves, which, to the best of our knowledge, have not been published before. The results on experienced work difficulties can provide valuable input for clinicians and for the development of interventions. A possible limitation of this study is the cross-sectional design, which does not allow any conclusions on causal relationships between paid employment and sick leave, and clinical factors, QoL and anxiety and depression. Furthermore, the fact that we included an outpatient population from a highly specialized referral hospital might have influenced the outcomes of the study because more patients with a poor prognosis visited our hospital. Earlier research has shown that patients with a poor prognosis have higher rates of anxiety and depression $[22,23]$, which in turn were associated with less favourable work outcomes in our study. Therefore, the rates of unemployment might have been higher in our sample than in other samples from other general hospitals. Another limitation might be the limited number of patients in the multiple analyses. We cannot exclude that some findings might be based on chance. However, the results were indicative of a general pattern and therefore underscore the general conclusion.

\section{Paid employment}

In our study, we found that $61 \%$ of IBD patients were in paid employment. In the total Dutch population aged 15 to 64 years, $68 \%$ performed paid work for at least $12 \mathrm{~h}$ per week in 2008 [24]. The employment rate among IBD patients in our sample appears to be slightly lower than that of the general Dutch population, although samples were not matched on age, sex and education, and results were not statistically analysed. It seems plausible that IBD patients have lower paid employment rates than the general population because of the physical, emotional and social problems that they experience including fatigue, pain, diarrhoea, embarrassment, anxiety or depression. Another possibility is the fact that young IBD patients tend to have less paid jobs during high school than their peers [25], a trend that persists after high school. We hypothesize that this lack of work experience is likely to influence the chances of successful employment for IBD patients after school. More attention to the careers of IBD patients at a very early stage might positively affect their chances of paid employment.

The work disability rate in our study was $23 \%$ compared with $10 \%$ in the general Dutch population [24]. Although samples were not matched on age, sex and education, disability rates of IBD patients in our sample seem to be relatively high. Earlier studies in Norway [8], Hungary [7] and the Netherlands [6] found disability rates of 19,32 and $18 \%$ (CD patients) and 10\% (UC patients), 
respectively. Hence, different work disability percentages are found in different countries and different cohorts. Differences in social policies, clinical and socioeconomic circumstances are likely to have influenced these work disability rates. Nonetheless, the results of different studies and countries uniformly indicate that the work disability rate of IBD patients is higher than that in the general population.

\section{Work difficulties}

A remarkable result was that almost half of the patients who were working reported that their work had been hindered by health problems in the last 2 weeks while other difficulties at work such as concentration problems and difficulties making decisions at work were frequent. It is likely that these difficulties experienced at work have a negative impact on the job opportunities and careers of IBD patients. These results are in line with an earlier survey of the Work Foundation [26] among 246 patients in the UK with a chronic disease including IBD patients $(28 \%)$, patients with diabetes $(13 \%)$ and patients with musculoskeletal disorders (11\%). A significant proportion of the respondents highlighted the negative impact that their chronic condition had on their careers [26], including not reaching their full potential in the workplace, inhibited career progression and lower job satisfaction. It is very likely that the IBD patients in our sample will similarly suffer from limited career prospects and work satisfaction.

An important adverse effect of IBD on functioning at work was presenteeism. In our study, the majority of patients reported that they were at work, but their work production was delayed, that they had to work slower than normal and/or that their work had to be done by others, which are all indicators of presenteeism. Presenteeism seems to be common among IBD employed patients because it was similarly reported in a Hungarian study in $60 \%$ of IBD patients, leading to a $28 \%$ loss of work productivity and a $32 \%$ activity loss [7]. Presenteeism can also have a severe negative impact on patients' life because it is associated with reduced QoL [27]. Moreover, IBD patients in our study without paid employment reported lower QoL and higher anxiety and depression rates than those in paid employment, which is in line with earlier research from Norway [5].

Interestingly, the sick leave rate in our patients was quite high, with $25 \%$ in the last 2 weeks. However, sick leave in our study was not associated with disease activity, which is in contrast to other recent research indicating that disease course was associated with work disability. [6,7]. It might be possible that disease activity does not influence sick leave because patients go to work irrespective of active disease, leading to presenteeism and ultimately to work disability. This possible mechanism is supported by the finding that not having paid employment was related to higher disease activity in our study.

\section{Healthcare utilization}

Visits to the occupational physician, GP, physiotherapist and medical specialist were highly associated with QoL measured by the IBDQ, and to a lesser extent, with the SF12 physical scale and anxiety. This finding confirms our earlier research in which we found that poor QoL, experienced disease burden and depression were significantly $(P<0.01)$ associated with more visits to both the gastroenterologist and the GP [28]. This could imply that not only the medical symptoms of IBD should be treated but also anxiety, depression and emotional and social difficulties resulting from having IBD. Furthermore, this indicates that the IBDQ could be used as a valuable instrument to detect symptoms and difficulties that are associated with higher healthcare utilization. Those patients with higher symptom burden and lower functioning should consequently receive more guidance and support to prevent those unfavourable outcomes.

\section{Implications for research and practice}

Most importantly, our study shows opportunities to solve the work-related difficulties encountered by the patients. Employment outcomes can be improved with innovations in treatment and with clinical and supportive services aimed at better management of symptoms and accommodation of disabilities [7]. Moreover, if patients keep participating in work life as much as possible, the chances of work retention increase significantly. Therefore, psychosocial interventions should be developed to enable IBD patients to solve their difficulties at work or to succeed in appropriate employment as early as possible. This can improve their social position, financial situation, QoL and prevent development of anxiety and depression symptomatology. Similar to other chronic conditions, these interventions could include multidisciplinary teams $[29,30]$ of clinicians and nurses.

Clinicians and nurses could also play an important role in detecting patients at risk because our study has shown that the indication of patients with possible work difficulties can be signalled in the clinic when IBD patients indicate that they have work-related problems. Clinicians and nurses could identify patients at risk for work difficulties very early in the treatment process and, if indicated, refer them to occupational professionals such as occupational physicians or occupational nurses to prevent workrelated problems and associated unfavourable outcomes.

\section{Conclusion}

More than half of IBD patients in our study were in paid employment, whereas almost a quarter was receiving a disability pension. A large majority of patients at work experienced difficulties such as concentration problems, having to work slower than normal, having to seclude themselves from their colleagues and a delay in the production of their work. Having no paid employment was associated with poorer QoL, and more anxiety and depression symptomatology.

\section{Acknowledgements}

\section{Conflicts of interest}

There are no conflicts of interest.

\section{References}

1 Burisch J, Jess T, Martinato M, Lakatos PL. ECCO-EpiCom. The burden of inflammatory bowel disease in Europe. J Crohns Colitis 2013; 7:322-337. 
2 Bennebroek Evertsz' F, Bockting CL, Stokkers PC, Hinnen C, Sanderman R, Sprangers MA. The effectiveness of cognitive behavioral therapy on the quality of life of patients with inflammatory bowel disease: multi-center design and study protocol (KL!C-study). BMC Psychiatry 2012; 12:227-227.

3 De Boer AG, Wijker W, Bartelsman JF, de Haes HC. Inflammatory Bowel Disease Questionnaire: cross-cultural adaptation and further validation. Eur J Gastroenterol Hepatol 1995; 7:1043-1050.

4 Molodecky NA, Soon IS, Rabi DM, Ghali WA, Ferris M, Chernoff G, et al. Increasing incidence and prevalence of the inflammatory bowel diseases with time, based on systematic review. Gastroenterology 2012; 142:46-54.

5 Bernklev T, Jahnsen J, Henriksen M, Lygren I, Aadland E, Sauar J, et al. Relationship between sick leave, unemployment, disability, and healthrelated quality of life in patients with inflammatory bowel disease. Inflamm Bowel Dis 2006; 12:402-412.

6 Van der Valk ME, Mangen MJ, Leenders M, Dijkstra G, van Bodegraven AA, Fidder $\mathrm{HH}$, et al. Risk factors of work disability in patients with inflammatory bowel disease - a Dutch nationwide webbased survey: work disability in inflammatory bowel disease. J Crohns Colitis 2014; 8:590-597.

7 Mandel MD, Bálint A, Lovász BD, Gulácsi L, Strbák B, Golovics PA, et al. Work disability and productivity loss in patients with inflammatory bowel diseases in Hungary in the era of biologics. Eur $\mathrm{J}$ Health Econ 2014; 15 (Suppl 1):S121-S128.

8 Høivik ML, Moum B, Solberg IC, Henriksen M, Cvancarova M, Bernklev T. IBSEN Group. Work disability in inflammatory bowel disease patients 10 years after disease onset: results from the IBSEN Study. Gut 2013; 62:368-375.

9 Siebert U, Wurm J, Gothe RM, Arvandi M, Vavricka SR, von Känel R, et al. Predictors of temporary and permanent work disability in patients with inflammatory bowel disease: results of the Swiss inflammatory bowel disease cohort study. Inflamm Bowel Dis 2013; 19:847-855.

10 Gunnarsson C, Chen J, Rizzo JA, Ladapo JA, Naim A, Lofland JH. The employee absenteeism costs of inflammatory bowel disease: evidence from US National Survey Data. J Occup Environ Med 2013; 55:393-401.

11 Vergara M, Montserrat A, Casellas F, Gallardo O, Suarez D, Motos J, et al. Development and validation of the Crohn's disease perceived work disability questionnaire. Inflamm Bowel Dis 2011; 17:2350-2357.

12 Varekamp I, Verbeek JH, de Boer A, van Dijk FJ. Effect of job maintenance training program for employees with chronic disease - a randomized controlled trial on self-efficacy, job satisfaction, and fatigue. Scand J Work Environ Health 2011; 37:288-297.

13 Nahon S, Lahmek P, Durance C, Olympie A, Lesgourgues B, Colombel JF, Gendre JP. Risk factors of anxiety and depression in inflammatory bowel disease. Inflamm Bowel Dis 2012; 18:2086-2091.

14 Hakkaart-Van Roijen L. Manual Trimbos/iMTA questionnaire for costs associated with psychiatric illness. J Consult Clin Psychol 2002; 59:12-19.

15 Harvey RF, Bradshaw JM. A simple index of Crohn's-disease activity. Lancet 1980; 1:514.
16 Truelove SC, Witts LJ. Cortisone in ulcerative colitis; preliminary report on a therapeutic trial. Br Med J 1954; 2:375-378.

17 Gandek B, Ware JE, Aaronson NK, Apolone G, Bjorner JB, Brazier JE, et al. Cross-validation of item selection and scoring for the SF-12 Health Survey in nine countries: results from the IQOLA Project. J Clin Epidemiol 1998; 51:1171-1178.

18 Ware JE, Kosinski M, Keller SD. SF-12: How to score the SF-12 physical and mental health summary scales, 3rd ed. Boston, MA: The Health Institute, New England Medical Center; 1998.

19 Guyatt G, Mitchell A, Irvine EJ, Singer J, Williams N, Goodacre R, Tompkins C. A new measure of health status for clinical trials in inflammatory bowel disease. Gastroenterology 1989; 96:804-810.

20 Cheung WY, Garratt AM, Russell IT, Williams JG. The UK IBDQ-a British version of the inflammatory bowel disease questionnaire. development and validation. J Clin Epidemiol 2000; 53:297-306.

21 Zigmond AS, Snaith RP. The hospital anxiety and depression scale. Acta Psychiatr Scand 1983; 67:361-370.

22 Bennebroek Evertsz' F, Thijssens NA, Stokkers PC, Grootenhuis MA, Bockting CL, Nieuwkerk PT, Sprangers MA. Do inflammatory bowel disease patients with anxiety and depressive symptoms receive the care they need? J Crohns Colitis 2012; 6:68-76.

23 Kim ES, Cho KB, Park KS, Jang BI, Kim KO, Jeon SW, et al. Predictive factors of impaired quality of life in Korean patients with inactive inflammatory bowel disease: association with functional gastrointestinal disorders and mood disorders. J Clin Gastroenterol 2013; 47:e38-e44.

24 Statistics Netherlands. Labour participation; 2011. Available at: $h$ ttp:// statline. cbs.n//StatWeb/publication/?VW $=T \& D M=S L N L \& P A=71738$ $N E D \& D 1=24-25 \& D 2=a \& D 3=0 \& D 4=0 \& D 5=6,11,16,21,26,31,36$, $41,46,51,60,65, / \& H D=140304-0958 \& H D R=G 1, T \& S T B=G 2, G 3, G 4$. [Accessed 4 March 2016].

25 Hummel TZ, Tak E, Maurice-Stam H, Benninga MA, Kindermann A, Grootenhuis MA. Psychosocial developmental trajectory of adolescents with inflammatory bowel disease. J Pediatr Gastroenterol Nutr 2013; 57:219-224

26 The Work Foundation. Management of long-term conditions. Available at: http://www.theworkfoundation.com/. [Accessed 4 March 2016].

27 Glanz BI, Dégano IR, Rintell DJ, Chitnis T, Weiner HL, Healy BC. Work productivity in relapsing multiple sclerosis: associations with disability, depression, fatigue, anxiety, cognition, and health-related quality of life (QoL). Value Health 2012; 15:1029-1035.

28 De Boer AG, Sprangers MA, Bartelsman JF, de Haes HC. Predictors of health care utilization in patients with inflammatory bowel disease: a longitudinal study. Eur J Gastroenterol Hepatol 1998; 10:783-789.

29 de Boer AG, Taskila TK, Tamminga SJ, Feuerstein M, FringsDresen $\mathrm{MH}$, Verbeek $\mathrm{JH}$. Interventions to enhance return-towork for cancer patients. Cochrane Database Syst Rev 2015; 9: CD007569.

30 Jensen C, Nielsen CV, Jensen OK, Petersen KD. Cost-effectiveness and cost-benefit analyses of a multidisciplinary intervention compared with a brief intervention to facilitate return to work in sicklisted patients with low back pain. Spine (Phila Pa 1976) 2013; 38:1059-1067. 